\title{
Motivación de estudiantes de ingeniería en informática con énfasis en ingeniería de software: un estudio en universidades latinoamericanas*
}

\author{
Raquel Anaya Hernández** \\ Marisa Cecilia Tumino** \\ Jhon Fredy Niño Manrique ${ }^{* * * *}$ \\ Juan Manuel Bournissen ${ }^{* * * * *}$ \\ Walter Hugo Arboleda Mazo ${ }^{* * * * * *}$
}

\author{
Recibido: 15/11/2019 • Aceptado: 03/04/2020 \\ https://doi.org/10.22395/rium.v19n36a12
}

\begin{abstract}
Resumen
El estudio de la motivación de los futuros profesionales de desarrollo de software es de vital importancia teniendo en cuenta el fenómeno de alta demanda no satisfecha de estos profesionales y la responsabilidad de la academia de propender por un proceso de enseñanza-aprendizaje significativo y activo que logre comprometer positivamente a los estudiantes. Los objetivos contemplados fueron: a) identificar los aspectos que inciden en la motivación de los estudiantes de informática con énfasis en el área de la ingeniería de software; b) comprobar la fiabilidad y validez del instrumento elaborado; c) identificar los niveles de motivación de la población estudiada. Para el logro de estos objetivos, se diseñó y validó un nuevo instrumento y se realizó el análisis de datos en dos fases para obtener una estructura de factores y subfactores que den cuenta del nivel de motivación. En la primera fase del análisis se obtuvo un modelo estadístico de cuatro factores (atributos de desempeño; relaciones con estudiantes y docentes; desempeño de los docentes; recursos físicos y virtuales) que explican el 71,5\% de la variabilidad. En la segunda fase del análisis se refinó el modelo para identificar los subfactores que lo componen, con lo que se logró un alto nivel de confiabilidad. En cuanto a atributos de desempeño, se evidencia la necesidad de ayudar a los estudiantes a manejar su tiempo; las estrategias pedagógicas muestran poder predictivo sobre la motivación de los estudiantes en el área de ingeniería de software. Se concluye que la estructura de factores y subfactores obtenida con un alto nivel de confiabilidad sirvió como punto de partida para identificar acciones de mejora en las instituciones autoras del artículo; la fiabilidad y validez del instrumento lo perfilan como candidato para ser aplicado en programas similares de otras instituciones.
\end{abstract}

Palabras clave: motivación de estudiantes; enseñanza de la ingeniería de software; perfil del estudiante de informática; perfil del desarrollador de software.

* Artículo derivado del proyecto de investigación Marco de referencia para la enseñanza de la ingeniería de
software en universidades adventistas de Latinoamérica, financiado por la Corporación Universitaria Adventista
en Colombia y la Universidad Adventista del Plata en Argentina.
Doctora en Informática, Sistemas Informáticos y Computación. Asesora de investigación, Grupo de Investigación
en Ingeniería Aplicada GI2A, Facultad de Ingeniería, Corporación Universitaria Adventista, Medellín, Colombia.
Correo electrónico: raquel.anaya.hdez@gmail.com. Orcid: https://orcid.org/0000-0002-9187-7427
Doctora en Educación con énfasis en Administración Educativa. Secretaria de Investigación, Facultad de
Ciencias Económicas y de la Administración, Universidad Adventista del Plata, Libertador San Martín,
Argentina. Correo electrónico: marisa.tumino@uap.edu.ar. Orcid: https://orcid.org/0000-0002-1913-6931
Magister en Ingeniería. Decano e investigador, Grupo de Investigación en Ingeniería Aplicada GI2A, Facultad
de Ingeniería, Corporación Universitaria Adventista, Medellín, Colombia. Correo electrónico: jfnino@unac.
edu.co. Orcid: https://orcid.org/0000-0003-3909-844X
Doctor Tecnologías Educativas: Educación Virtual y Gestión del Conocimiento. Docente e investigador,
Sistema de Educación a Distancia, Universidad Adventista del Plata, Libertador San Martín, Argentina. Correo
electrónico: juan.bournissen@uap.edu.ar. Orcid: https://orcid.org/0000-0002-8246-927X
Magíster en Ingeniería. Investigador, Grupo de Investigación en Ingeniería Aplicada GI2A, Facultad de
Ingeniería, Corporación Universitaria Adventista. Correo electrónico: warboleda@unac.edu.co. Orcid: https://
orcid.org/0000-0003-4937-5359 


\title{
Motivation of Informatics Engineering Students with Emphasis on Software Engineering: a Study in Latin-American Universities
}

\begin{abstract}
The study of the motivation of the future software engineers is of the uttermost importance taking into account the high demand unsatisfied by these professionals and the responsibility of the academy of promoting for a significative and active learning-teaching process that achieves positively compromised students. The contemplated objectives were: a) identifying the aspects related in the motivation of informatics students with an emphasis in the software engineering area; b) ascertaining the reliability and validity of the elaborated instrument; c) identifying the motivation levels of the studied population. For achieving these objectives, a new instrument was designed and validated, a two-phase data analysis was also performed for obtaining a factors and subfactors structure that gives account of the motivational level. In the first phase of the analysis we obtained a four factor statistic model (performance attributes, relationship between students and teacher; performance of the teachers; physical and virtual resources) that explain the $71,5 \%$ of variability. In the second phase of analysis the model for identifying the factors was refined and with that a high level of reliability was achieved. Regarding the performance attributes, we evidences a necessity for helping students to handle their own time; the pedagogic strategies display a high predictive power on the motivation of the students in the software engineering area. This article concludes that the factors and subfactors structures structures obtained with a high level of reliability served as a starting point for identifying improvement actions in the institutions responsible for the writing of the articles; the reliability and validity of this instrument profile it as a candidate for being applied in similar programs for other institutions.
\end{abstract}

Keywords: students motivation; software engineering teaching; informatics student profile; profile of the software developer.

\section{Motivação de estudantes de engenharia em informática com ênfase em engenharia de software: um estudo em universidades latino-americanas}

\begin{abstract}
Resumo
O estudo da motivação dos futuros profissionais de desenvolvimento de software é fundamental, tendo em vista o fenômeno da alta demanda não satisfeita desses profissionais e a responsabilidade da academia visar a um processo de ensino-aprendizagem significativo e ativo que consiga comprometer positivamente os estudantes. Nesse sentido, os objetivos deste estudo foram: a) identificar os aspectos que influenciam a motivação dos estudantes de informática com ênfase na área da Engenharia de Software; b) comprovar a confiabilidade e validade do instrumento elaborado; c) identificar os níveis de motivação da população estudada. Para atingi-los, um novo instrumento foi elaborado e testado, e a análise de dados foi realizada em duas fases para obter uma estrutura de fatores e subfatores que evidenciem o nível de motivação. Na primeira fase da análise, foi obtido um modelo estatístico de quatro fatores (atributos de desempenho; relações com estudantes e docentes; desempenho dos docentes; recursos físicos e virtuais) que explicam $71,5 \%$ da variabilidade. Na segunda, o modelo foi aprimorado para identificar os subfatores que o compõem; com isso, conseguiu-se um alto nível de confiabilidade. Quanto aos atributos de desempenho, verificou-se a necessidade de ajudar os estudantes a lidar com seu tempo; as estratégias pedagógicas mostram poder preditivo sobre a motivação dos estudantes na área de Engenharia de Software. Conclui-se que a estrutura de fatores e subfatores obtida com um alto nível de confiabilidade serviu como ponto de partida para identificar ações de melhoria nas instituições autoras deste artigo; a confiabilidade e a validade do instrumento o caracterizam como candidato para ser aplicado em programas similares de outras instituições.
\end{abstract}

Palavras-chave: motivação de estudantes; ensino da Engenharia de Software; perfil do estudante de informática; perfil do desenvolvedor de software. 


\section{INTRODUCCIÓN}

El desarrollo acelerado de las tecnologías de información y comunicación (TIC) crea oportunidades y retos importantes para las carreras de ingeniería en informática en Latinoamérica. En el estudio de carreras de informática realizado por el proyecto Tunning de Latinoamérica [1], se aplicó una encuesta a empleadores, graduados, académicos y estudiantes de países de Latinoamérica. Se encontró que las tres competencias más importantes en los programas de ingeniería de sistemas o de informática están directamente relacionadas con el desarrollo de soluciones informáticas desde su concepción, diseño, desarrollo y operación usando estándares de calidad.

El papel de la academia es clave para dinamizar la industria de software [2]. Por tanto, una adecuada formación en el campo de la ingeniería de software es uno de los retos de la universidad latinoamericana. El objetivo de esta formación es responder a situaciones, tales como el interés de los gobiernos latinoamericanos por promover la industria de software como un renglón importante de la economía de su países, reconocida como un "sector de clase mundial" [3]; crear alianzas con diferentes sectores que promueven la creación de servicios de alto valor agregado [4]; corresponder a la alta demanda de profesionales de software y el papel protagónico del talento humano como ventaja competitiva de la industria de software [5].

La motivación es una de las claves para el desempeño académico de los estudiantes universitarios [6]. Las carreras de ingeniería frecuentemente presentan altos niveles de deserción y, por tanto, se requieren estrategias que la disminuyan y que contribuyan a la formación adecuada de los estudiantes como profesionales [7-8].

Este trabajo retoma las definiciones de motivación citadas por Murphy y Alexander [9]. La motivación es "el proceso psicológico involucrado en la dirección, el vigor y persistencia del comportamiento" [10]. También se hace una distinción entre motivación intrínseca y extrínseca [11]; mientras que la motivación intrínseca es definida como "una tarea que se realiza porque es gratificante en sí misma debido a una recompensa que se gana como consecuencia", la motivación extrínseca se define como "realizar una tarea para obtener algo fuera de la actividad en sí". La distinción entre estos dos tipos de motivaciones servirá de punto de partida en este trabajo para la identificación de la estructura de factores y subfactores: mientras que en la primera categoría se agrupan aquellas motivaciones que son inherentes al aprendiz, en la segunda categoría se agrupan aspectos de motivación que se generar desde el contexto.

La motivación de los estudiantes es ampliamente tratada en la literatura. Una premisa generalizada es que la motivación es esencial para favorecer el aprendizaje de los estudiantes. Pintrich [12] propone un marco de trabajo para evaluar la motivación y 
el aprendizaje del estudiante, soportado en la perspectiva de aprendizaje autorregulado. Por otra parte, el trabajo de Álvarez et al. [13] comprobó que una motivación adecuada y una revisión de los métodos de evaluación utilizados pueden favorecer el logro del aprendizaje significativo.

El proceso de enseñanza-aprendizaje puede ser entendido desde la teoría de la actividad [14-15], en la cual los estudiantes construyen gradualmente el conocimiento a partir de la actividad que los relaciona con los objetos del mundo (el contexto) al interactuar con los demás. Leontiev [14] define actividad como el conjunto de acciones que presentan una motivación intrínseca del sujeto, que lo conduce a desarrollarlas activamente para que el proceso no quede solo en un nivel operativo y mecánico.

Como marco conceptual para el presente estudio, se toman trabajos previos [16-17] que definen un modelo de enseñanza en el contexto de la ingeniería de software basado en la teoría de la actividad con tres dimensiones: aprendiz, cuerpo de conocimiento y contexto. Entre estos elementos se suceden relaciones de tensión. El aprendiz debe atender a múltiples demandas: a) las solicitadas por el entorno cultural e industrial que le reclama vínculos coherentes con el conocimiento colectivo específico hacia la ingeniería de software; b) las que le solicita el objeto de aprendizaje (ingeniería de software); $\mathrm{y}$ c) las motivaciones y competencias que tiene como aprendiz para responder a las demandas del contexto (la industria de software) como de la naturaleza del objeto de conocimiento (la ingeniería de software).

El objetivo de este trabajo es identificar, validar y analizar los factores que inciden en la motivación de los estudiantes de informática en general y, específicamente, la motivación por el área de la ingeniería de software de los estudiantes de trece universidades ubicadas en siete países de Latinoamérica que se sustentan en una cosmovisión cristiana integral [18]. Para tal propósito, se diseñó un instrumento a partir del trabajo de Fernández et al. [6]. Estos autores proponen un instrumento diagnóstico basado en seis teorías de motivación humana aplicadas en el campo de la psicología del trabajo conformado por cuarenta y ocho ítems. También se tuvo en cuenta el trabajo de Bechamn et al. [19], quienes realizaron una revisión sistemática de estudios existentes de motivación en desarrolladores e identificaron las características de los ingenieros de software. Por último, se consideró el trabajo de Hall et al. [20] que identifica los motivadores generales de los desarrolladores de software.

El artículo se estructura en cuatro secciones. En la primera sección se describen las actividades, materiales y métodos utilizados en las diferentes fases del estudio. En la segunda sección se presentan los resultados. En la tercera sección se realiza la discusión y acciones de mejora que se están llevando a cabo. Por último, se presentan las conclusiones. 


\section{MATERIALES Y MÉTODOS}

En el estudio participaron cuatrocientos ocho estudiantes de informática, distribuidos en trece universidades de siete países latinoamericanos. Luego del estudio de los referentes, se diseñó un instrumento nuevo porque se consideró que ninguno de los instrumentos existentes cumplía con los objetivos definidos de perfilar el nivel de motivación de los estudiantes en general que a la vez permitiera identificar aspectos de motivación particulares del área de ingeniería de software.

\subsection{Diseño de la encuesta}

Para el diseño de la encuesta se asumió que la teoría del factor dual de Herzberg, que distingue los aspectos motivacionales intrínsecos y extrínsecos y que fue adaptada por Fernández et al. [6] al contexto académico, era un buen referente de partida para identificar los aspectos de motivación relevantes que permitirían evaluar el nivel de motivación en los estudiantes.

Los aspectos intrínsecos (IN) identificados en las preguntas correspondientes del instrumento encuesta aplicado fueron: orientación al logro (preguntas 11, 12, 18, 29, 30, 31,33 y 34), responsabilidad (preguntas 35 y 36), formación integral (preguntas 10 y 20), creatividad (preguntas 26 y 27), competencia técnica (preguntas 17 y 19) e identificación con la tarea como ingeniero de software (preguntas 21, 23, 24, 25, 32, 39 y 40).

Los aspectos extrínsecos (EX) considerados fueron: calidad de la docencia (preguntas 7, 8, 9, 37, 38 y 22), relación con estudiantes y docentes (preguntas 5, 13, $15,16,28,6$ y 14) y recursos físicos y virtuales (preguntas $1,2,3$ y 4). Si bien se parte de estos aspectos iniciales, es importante aclarar que en el instrumento no se hizo una identificación explícita de las categorías o aspectos, con el fin de evitar cualquier tipo de sesgo en los encuestados, además de buscar que el modelo de factores surgiera a partir del análisis estadístico.

La primera versión del instrumento fue sometida a una validación de contenido por parte de cinco expertos: cuatro docentes del programa de informática de las diferentes universidades involucradas y un colaborador externo experto en el tema de ingeniería de software experimental. Cada experto hizo lectura de la encuesta y dio su retroalimentación en cada ítem de esta, con respecto a la pertinencia y la claridad. Estos aspectos fueron evaluados en una escala de uno a cinco y se acompañaron con observaciones y recomendaciones. Una vez introducidas las modificaciones, el instrumento contó con cuarenta ítems evaluados en una escala tipo Likert de cinco niveles con sus correspondientes equivalencias cuantitativas: totalmente en desacuerdo $(\mathrm{TD}=1)$; en desacuerdo $(\mathrm{D}=2)$; de acuerdo $(\mathrm{A}=4)$; totalmente de acuerdo $(\mathrm{TA}=5)$; y no aplica $(\mathrm{NA}=3)$. 
La encuesta se desarrolló usando Google Forms, exceptuando una institución que lo aplicó presencialmente. La encuesta fue respondida entre el 21 de junio y el 15 de noviembre de 2016. Los docentes de cada institución motivaron a sus estudiantes a responder la encuesta de manera voluntaria.

\subsection{Tratamiento inicial de los datos}

Se hizo un análisis descriptivo de las variables de género, edad y universidad de origen, y se procedió a los análisis respectivos. A continuación, se realizó un análisis factorial exploratorio para medir la idoneidad de los datos utilizando la prueba de KMO (Kaiser-Meyer-Olkin). Asimismo, se realizó un análisis de factores rotados para obtener el modelo de agrupación de factores utilizando Varimax. Para cada uno de los factores obtenidos, se analizó la consistencia interna de las escalas mediante el Alfa de Cronbach. Se aplicó luego un análisis de regresión lineal múltiple con el método por pasos sucesivos para identificar el poder predictor de las variables.

Con el propósito de analizar el comportamiento de las dimensiones de motivación obtenidas según las variables categóricas que participaron del estudio, se procedió a aplicar pruebas inferenciales con las variables género, institución y nivel académico.

El nivel académico se definió de acuerdo con el semestre que cursaba el estudiante: básico (1), entre primer y tercer semestre; intermedio (2), entre cuarto y séptimo semestre; y el nivel avanzado (3), de octavo en adelante.

\subsection{Tratamiento posterior de los datos}

En la siguiente fase del análisis, se partió del modelo de factores obtenido en la primera fase para explicitar un modelo de subfactores, con el propósito de analizar, de manera más precisa, el comportamiento de estos en el contexto de la ingeniería de software. Mediante el Alfa de Cronbach, se identificó la consistencia interna de la escala de subfactores y se procedió a analizar su comportamiento con respecto al nivel académico. El análisis estadístico fue realizado mediante el programa SPSS (Statistical Package for Social Sciences) para Windows, versión 15.0.

\section{RESULTADOS}

\subsection{Análisis descriptivo}

La muestra de los cuatrocientos ocho estudiantes estuvo conformada por trescientos treinta y dos hombres $(81 \%)$ y setenta y seis mujeres (19\%) con una media de edades de veintiún años, y la moda y la mediana se ubicaron en los veinte años. La distribución de los encuestados por universidad se detalla en la tabla 1. 
Tabla 1. Distribución de frecuencias entre las diferentes instituciones participantes

\begin{tabular}{ccc}
\hline Instituciones & Frecuencia & Porcentaje \\
\hline UN1 & 30 & 7,4 \\
\hline UN2 & 17 & 4,2 \\
\hline UN3 & 12 & 2,9 \\
\hline UN4 & 51 & 12,5 \\
\hline UN5 & 19 & 4,7 \\
\hline UN6 & 59 & 14,5 \\
\hline UN7 & 39 & 9,6 \\
\hline UN8 & 99 & 24,3 \\
\hline UN9 & 8 & 2,0 \\
\hline UN10 & 8 & 2,0 \\
\hline UN11 & 13 & 3,2 \\
\hline UN12 & 53 & 13,0 \\
\hline UN13 & 1 & 0,2 \\
\hline Total & 408 & 100
\end{tabular}

Fuente: elaboración propia.

\subsection{Validación de la escala de motivación}

El análisis factorial exploratorio de los cuarenta ítems de la escala mostró un KMO de 0,978 , lo que indica la adecuación muestral para aplicar el análisis. La matriz de factores rotados, utilizando la rotación Varimax, mostró un modelo de cuatro factores que explican el 71,5\% de la variabilidad de las variables, lo que indica un buen ajuste del modelo. La saturación de las variables en los cuatro factores agrupó los siguientes ítems o preguntas:

- Factor 1, compuesto por los ítems 11, 12, 17, 18, 19, 20, 21, 23, 24, 25, 26, 27, 29, 30, $31,32,33,34,35,36,37,38,39$ y 40 , representa los atributos de desempeño propios del estudiante.

- Factor 2, compuesto por los ítems 5, 6, 10, 13, 14, 15, 16, 22 y 28, representa las relaciones con estudiantes y docentes.

- Factor 3, compuesto por los ítems 7, 8 y 9, representa las estrategias pedagógicas aplicadas por los docentes.

- Factor 4, compuesto por los ítems 1,2, 3 y 4, representa los recursos físicos y virtuales. 
Para validar la consistencia interna de las escalas, se analizó la confiabilidad mediante el Alfa de Cronbach. Los ítems asociados al factor 1 arrojaron un Alfa de Cronbach de 0,981. Los correspondientes al factor 2 mostraron un valor de 0,928. Los ítems del factor 3 obtuvieron un valor de 0,869. Finalmente, los ítems del factor 4 alcanzaron un 0,821. En todos los casos, los análisis no sugirieron la eliminación de ítems con el propósito de incrementar el valor del estadístico de fiabilidad.

\subsection{Análisis de regresión lineal}

Para identificar el poder predictor de las variables de los factores 2, 3 y 4 sobre la variable del factor 1 , se aplicó un análisis de regresión lineal múltiple con el método por pasos sucesivos, donde las variables predictoras 2, 3 y 4 actuaron como variables de entrada y el factor 1 actuó como variable dependiente.

Se encontró una relación significativa entre las variables (ecuación 1).

Ecuación 1. Ecuación predictora del desempeño del estudiante

$$
Y=0,102+0,782 X 1+0,111 X 2+0,071 X 3
$$

Donde $Y$ representa los atributos de desempeño, $X 1$ las relaciones entre estudiantes y docentes, $X 2$ el desempeño de los docentes y $X 3$ los recursos físicos y virtuales.

El coeficiente de determinación (R2) fue de 0,832 , lo que revela un gran poder predictor de las variables de entrada sobre los atributos de desempeño de los estudiantes, siendo las relaciones entre estudiantes y docentes la variable con mayor ponderación. El error cuadrático medio fue de 0,16. En la tabla 2 se muestran los coeficientes tipificados y los valores de probabilidad asociados, indicando en todos los casos significación estadística.

Tabla 2. Coeficientes tipificados y valores de probabilidad $(n=408)$

\begin{tabular}{lccc}
\hline & $B$ & $S E \beta$ & $\begin{array}{c}B \\
\text { estandarizado }\end{array}$ \\
\hline Constante & 0,102 & 0,090 & \\
\hline Relaciones & 0,828 & 0,032 & $0,782^{*}$ \\
\hline Estrategias pedagógicas & 0,109 & 0,029 & $0,111^{*}$ \\
\hline Recursos & 0,066 & 0,030 & $0,071^{*}$ \\
\hline
\end{tabular}

* Indica que el valor del coeficiente es significativamente diferente de $0(\mathrm{p}<0,05)$

Fuente: elaboración propia. 


\subsection{Comparación de medias de dimensiones}

Para analizar el comportamiento de los factores de motivación según las variables categóricas que participaron del estudio (institución, género y nivel de formación), se aplicaron pruebas inferenciales.

- Motivación con respecto a instituciones participantes y con respecto a género. Al aplicar el análisis de varianza de un factor con el propósito de identificar diferencias de medias de las dimensiones de motivación entre los grupos definidos por las instituciones participantes, no se observaron diferencias estadísticamente significativas $(\mathrm{p}>0,05)$. De manera similar, la prueba $T$ para dos muestras independientes no mostró suficiente evidencia empírica para inferir diferencias significativas en las dimensiones de motivación entre mujeres y hombres $(\mathrm{p}>0,05)$.

- Motivación y nivel académico. Se pretendió inferir el comportamiento del nivel académico de los estudiantes en el nivel de motivación percibido para los cuatro factores resultantes. Para ello, se utilizó la variable categorizada de nivel académico como factor intersujeto en el análisis multivariante del modelo lineal general. Se observa que existe un efecto principal significativo del nivel académico, $\mathrm{F}(2,377)=3,18, \mathrm{p}<0,05, \eta 2=0,017$, en el nivel de motivación correspondiente a los recursos físicos y virtuales. Existen diferencias estadísticamente significativas en las medias de motivación vinculada a los recursos físicos y virtuales $(\mathrm{p}<0,05)$ entre los estudiantes del nivel académico avanzado $(\mathrm{M}=3,44$, $\mathrm{DT}=1,12)$ y aquellos que cursan el nivel básico $(\mathrm{M}=3,78, \mathrm{DT}=0,99)$. Ese efecto no se presenta en los restantes factores.

\subsection{Análisis de subfactores para el perfil como desarrollador de software}

A partir del modelo de factores obtenido en la primera fase, se hizo una descomposición en subfactores con el propósito de explorar el comportamiento estadístico de aspectos que se consideran relevantes en los desarrolladores de software. Las tablas 3 a 6 presentan los respectivos factores con la propuesta de subfactores y los ítems que los conforman. Para cada ítem se presenta la media, la desviación estándar y el nivel interno de confiabilidad de la escala dada por el Alfa de Cronbach. En todos los subfactores se observa un alto nivel de confiabilidad de la escala. A continuación, se hace un análisis cualitativo de los resultados obtenidos en cada uno de los subfactores.

En la tabla 3 se puede observar que el subfactor 1.1 presenta resultados positivos (media promedio 4,1). Se destacan las características que se encuentran por encima del promedio: los estudiantes de ingeniería informática de las universidades involucradas en el estudio valoran la sensación de logro cuando consiguen realizar un trabajo o práctica 
exitosamente (30), cuando aprenden algo nuevo o adquieren una nueva habilidad (29) y cuando aprueban una asignatura con buenas notas (31); les gusta enfrentarse a actividades retadoras (34) y son conscientes de su responsabilidad en su proceso de aprendizaje $(35,36)$. De manera contrastante, el subfactor 1.3 (media promedio 3,9) identifica retos abiertos en los cuales deben seguir trabajando las diferentes instituciones para lograr fortalecer el perfil del estudiante de informática como ingeniero de software en los siguientes aspectos: propender por espacios de práctica en los que el estudiante pueda orientar su trabajo en actividades relacionadas con el desarrollo de software (25); involucrarse de manera activa en la planificación y gestión de los proyectos en los cuales participa (25); lograr que las prácticas pedagógicas aplicadas por los docentes del área de ingeniería de software sean altamente significativas para que contribuyan a su formación profesional (37); fomentar la motivación del estudiante hacia el área de ingeniería de software (39); y perfilarlo como un desarrollador exitoso de la industria (40). Por su parte, el subfactor 1.2 (media promedio 3,99) identifica oportunidades de mejora: la necesidad de una adecuada administración del tiempo del estudiante para fomentar su formación integral (20), el fortalecimiento de espacios que enamoren a los estudiantes con la creatividad $(26,27)$ y la importancia de buscar alta competencia técnica (17).

Tabla 3. Resultados del factor atributos de desempeño

\begin{tabular}{|c|c|c|c|c|}
\hline \multicolumn{5}{|c|}{ Factor 1: Atributos de desempeño } \\
\hline Subfactor & Ítems & Media & $\begin{array}{c}\text { Desviación } \\
\text { estándar }\end{array}$ & $\begin{array}{c}\text { Alfa de } \\
\text { Cronbach }\end{array}$ \\
\hline \multirow{6}{*}{$\begin{array}{l}\text { 1.1 Disposición } \\
\text { intrínseca hacia el } \\
\text { aprendizaje (DIA) }\end{array}$} & $\begin{array}{l}\text { 18. Oriento mi desempeño al logro de } \\
\text { objetivos. }\end{array}$ & 4,03 & 1,11 & 0,968 \\
\hline & $\begin{array}{l}\text { 23. Me sentiría bien al contribuir con el } \\
\text { desarrollo de la organización donde me } \\
\text { desempeñe en el futuro. }\end{array}$ & 4,09 & 1,16 & \\
\hline & $\begin{array}{l}\text { 29. Valoro la sensación de logro que tengo } \\
\text { cuando consigo aprender algo nuevo o } \\
\text { adquiero una nueva habilidad. }\end{array}$ & 4,16 & 1,12 & \\
\hline & $\begin{array}{l}\text { 30. Valoro la sensación de logro que } \\
\text { tengo cuando consigo realizar un trabajo } \\
\text { o una práctica exitosamente. }\end{array}$ & 4,18 & 1,13 & \\
\hline & $\begin{array}{l}\text { 31. Valoro la sensación de logro que tengo } \\
\text { cuando consigo aprobar una asignatura } \\
\text { con una buena calificación. }\end{array}$ & 4,15 & 1,18 & \\
\hline & $\begin{array}{l}\text { 33. Disfruto de la sensación de progreso } \\
\text { que obtengo cursando mis estudios. }\end{array}$ & 4,03 & 1,22 & \\
\hline
\end{tabular}


Factor 1: Atributos de desempeño

\begin{tabular}{|c|c|c|c|c|}
\hline Subfactor & Ítems & Media & $\begin{array}{l}\text { Desviación } \\
\text { estándar }\end{array}$ & $\begin{array}{c}\text { Alfa de } \\
\text { Cronbach }\end{array}$ \\
\hline \multirow{5}{*}{$\begin{array}{l}\text { 1.1 Disposición } \\
\text { intrínseca hacia el } \\
\text { aprendizaje (DIA) }\end{array}$} & $\begin{array}{l}\text { 34. Considero que estudiar es una } \\
\text { oportunidad para desarrollarme con } \\
\text { actividades retadoras. }\end{array}$ & 4,15 & 1,15 & \\
\hline & $\begin{array}{l}\text { 35. Considero que soy el principal } \\
\text { responsable de mi proceso de aprendizaje. }\end{array}$ & 4,10 & 1,22 & \\
\hline & $\begin{array}{l}\text { 36. Considero que soy el principal res- } \\
\text { ponsable de mis resultados académicos. }\end{array}$ & 4,13 & 1,23 & \\
\hline & $\begin{array}{l}\text { 38. Las actividades de clase y trabajos } \\
\text { prácticos me preparan para mi vida } \\
\text { profesional. }\end{array}$ & 4,03 & 1,20 & \\
\hline & Subtotal & 4,10 & 1,17 & \\
\hline \multirow{8}{*}{$\begin{array}{l}\text { 1.2 Disposición para } \\
\text { atender las demandas } \\
\text { del contexto (DDC) }\end{array}$} & $\begin{array}{l}\text { 11. Me siento bien al desarrollarme y } \\
\text { superarme con tareas difíciles. }\end{array}$ & 4,02 & 1,17 & \multirow{8}{*}{0,939} \\
\hline & $\begin{array}{l}\text { 12. Busco la excelencia en las tareas que } \\
\text { desempeño. }\end{array}$ & 4,01 & 1,20 & \\
\hline & $\begin{array}{l}\text { 17. Me preocupo por ser técnicamente } \\
\text { competente. }\end{array}$ & 4,00 & 1,22 & \\
\hline & $\begin{array}{l}\text { 19. Me gustan los trabajos prácticos que } \\
\text { me desafían a adquirir nuevas competen- } \\
\text { cias técnicas. }\end{array}$ & 4,05 & 1,19 & \\
\hline & $\begin{array}{l}\text { 20. Oriento la administración de mi } \\
\text { tiempo al crecimiento personal y a la } \\
\text { formación profesional. }\end{array}$ & 3,82 & 1,17 & \\
\hline & $\begin{array}{l}\text { 26. Me gusta ser creativo en los retos de } \\
\text { la vida diaria. }\end{array}$ & 4,00 & 1,19 & \\
\hline & $\begin{array}{l}\text { 27. Me agrada desarrollar los trabajos } \\
\text { prácticos que incentivan mis propias } \\
\text { ideas y me permiten aplicarla. }\end{array}$ & 4,07 & 1,12 & \\
\hline & Subtotal & 3,99 & 1,18 & \\
\hline \multirow{3}{*}{$\begin{array}{l}\text { 1.3 Disposición del } \\
\text { estudiante hacia el } \\
\text { objeto de conoci- } \\
\text { miento (DOC) }\end{array}$} & $\begin{array}{l}\text { 21. Me gusta participar en la planificación } \\
\text { de los trabajos prácticos del área de } \\
\text { software. }\end{array}$ & 3,81 & 1,18 & \multirow{3}{*}{0,929} \\
\hline & $\begin{array}{l}\text { 24. En el futuro quisiera crear mi propio } \\
\text { emprendimiento. }\end{array}$ & 4,07 & 1,28 & \\
\hline & $\begin{array}{l}\text { 25. Distribuyo mi tiempo entre mis } \\
\text { estudios y un trabajo/hobby relacionado } \\
\text { con mi profesión futura. }\end{array}$ & 3,75 & 1,25 & \\
\hline
\end{tabular}


Factor 1: Atributos de desempeño

\begin{tabular}{|c|c|c|c|c|}
\hline Subfactor & Items & Media & $\begin{array}{c}\text { Desviación } \\
\text { estándar }\end{array}$ & $\begin{array}{c}\text { Alfa de } \\
\text { Cronbach }\end{array}$ \\
\hline \multirow{5}{*}{$\begin{array}{l}\text { 1.3 Disposición del } \\
\text { estudiante hacia el } \\
\text { objeto de conoci- } \\
\text { miento (DOC) }\end{array}$} & $\begin{array}{l}\text { 32. Disfruto de la sensación de progreso } \\
\text { que obtengo cursando mis estudios. }\end{array}$ & 4,00 & 1,18 & \\
\hline & $\begin{array}{l}\text { 37. Las prácticas pedagógicas aplicadas } \\
\text { por los docentes en el área de ingeniería } \\
\text { de software contribuyen a mi formación } \\
\text { profesional. }\end{array}$ & 3,91 & 1,17 & \\
\hline & $\begin{array}{l}\text { 39. Me entusiasman las asignaturas } \\
\text { relacionadas con la ingeniería de } \\
\text { software. }\end{array}$ & 3,91 & 1,24 & \\
\hline & $\begin{array}{l}\text { 40. En mi futura vida profesional me } \\
\text { gustaría desempeñarme en actividades } \\
\text { directamente relacionadas con el } \\
\text { desarrollo de software. }\end{array}$ & 3,89 & 1,28 & \\
\hline & Subtotal & 3,90 & 1,22 & \\
\hline
\end{tabular}

Fuente: elaboración propia.

En el factor 2, presentado en la tabla 4, el subfactor 2.2 muestra un balance positivo en la percepción de los estudiantes con respecto a sus relaciones con los docentes (promedio 4,01). Por otro lado, en el subfactor 2.1 se identifican retos abiertos en la percepción de los estudiantes con respecto a las relaciones con sus compañeros de clase (promedio 3,91), especialmente en lo relacionado a la limitación en tiempo para hacer vida personal (10), y una relativamente baja percepción de su papel como influencia positiva en el aprendizaje de los compañeros (13), en su nivel de sociabilidad y disfrute del trabajo en equipo $(16,28)$.

Tabla 4. Resultados del factor relación con estudiantes y docentes

\begin{tabular}{|c|c|c|c|c|}
\hline \multicolumn{5}{|c|}{ Factor 2: Relación con estudiantes y docentes } \\
\hline Subfactor & İtems & Media & $\begin{array}{l}\text { Desviación } \\
\text { estándar }\end{array}$ & $\begin{array}{c}\text { Alfa de } \\
\text { Cronbach }\end{array}$ \\
\hline \multirow{4}{*}{$\begin{array}{l}2.1 \text { Relación } \\
\text { con compa- } \\
\text { ñeros (RCO) }\end{array}$} & $\begin{array}{l}\text { 5. Las relaciones académicas con mis compañeros } \\
\text { de clase son buenas. }\end{array}$ & 4,02 & 1,19 & \multirow{4}{*}{0,882} \\
\hline & $\begin{array}{l}\text { 10. El número de horas que me demandan las } \\
\text { actividades académicas en la universidad me } \\
\text { permite hacer vida personal. }\end{array}$ & 3,70 & 1,22 & \\
\hline & $\begin{array}{l}\text { 13. Influyo positivamente en el aprendizaje de } \\
\text { mis compañeros. }\end{array}$ & 3,85 & 1,14 & \\
\hline & $\begin{array}{l}\text { 15. Mantengo relaciones personales amistosas } \\
\text { con mis compañeros de clase. }\end{array}$ & 4,06 & 1,11 & \\
\hline
\end{tabular}


Factor 2: Relación con estudiantes y docentes

\begin{tabular}{|c|c|c|c|c|}
\hline Subfactor & İtems & Media & $\begin{array}{l}\text { Desviación } \\
\text { estándar }\end{array}$ & $\begin{array}{l}\text { Alfa de } \\
\text { Cronbach }\end{array}$ \\
\hline \multirow{3}{*}{$\begin{array}{l}2.1 \text { Relación } \\
\text { con compa- } \\
\text { ñeros (RCO) }\end{array}$} & $\begin{array}{l}\text { 16. Disfruto del trabajo en equipo con mis } \\
\text { compañeros. }\end{array}$ & 3,90 & 1,20 & \\
\hline & $\begin{array}{l}\text { 28. Soy sociable y me identifico con los compañeros } \\
\text { de los grupos en los que participo. }\end{array}$ & 3,92 & 1,19 & \\
\hline & Subtotal & 3,91 & 1,17 & \\
\hline \multirow{4}{*}{$\begin{array}{l}2.2 \text { Relación } \\
\text { con docentes } \\
\text { (RDO) }\end{array}$} & $\begin{array}{l}\text { 6. Las relaciones académicas con mis docentes } \\
\text { son buenas. }\end{array}$ & 4,04 & 1,16 & \multirow{4}{*}{0,857} \\
\hline & $\begin{array}{l}\text { 14. Me gusta obtener el reconocimiento de mis } \\
\text { profesores. }\end{array}$ & 3,90 & 1,22 & \\
\hline & $\begin{array}{l}\text { 22. Me gusta recibir retroalimentación de los } \\
\text { instructores por el trabajo realizado. }\end{array}$ & 4,09 & 1,17 & \\
\hline & Subtotal & 4,01 & 1,18 & \\
\hline
\end{tabular}

Fuente: elaboración propia.

La percepción del estudiante con respecto al factor 3 (estrategias pedagógicas en el área específica de ingeniería de software) presenta el segundo promedio más bajo de los resultados generales (promedio 3,82), tal como puede observarse en la tabla 5. Todos los ítems de este factor tienen un promedio por debajo de 4 , lo que indica que hay oportunidades de mejora importantes en las prácticas pedagógicas aplicadas por los docentes para lograr que estimulen el aprendizaje de la ingeniería de software (8), realicen una supervisión adecuada del trabajo tanto dentro como fuera de clase (9) y sepan compartir de forma adecuada sus conocimientos (7).

Tabla 5. Resultados del factor estrategias pedagógicas

\begin{tabular}{lllll}
\hline \multicolumn{5}{c}{ Factor 3: Estrategias pedagógicas } \\
\hline \multicolumn{1}{c}{ Subfactor } & \multicolumn{1}{c}{ Ítems } & Media & $\begin{array}{c}\text { Desviación } \\
\text { estándar }\end{array}$ & $\begin{array}{c}\text { Alfa de } \\
\text { Cronbach }\end{array}$ \\
\hline & $\begin{array}{l}\text { 7. Los profesores del área de ingeniería de software } \\
\text { conocen bien las materias que imparten y saben } \\
\text { cómo enseñármelas. }\end{array}$ & 3,89 & \multirow{2}{*}{0,869} \\
\cline { 2 - 5 } $\begin{array}{l}\text { 3.1 Es- } \\
\text { trategias } \\
\text { pedagógicas } \\
\text { (EPE) }\end{array}$ & $\begin{array}{l}\text { 8. Los profesores del área de ingeniería de software } \\
\text { conocen bien como motivarme, sus estilos y } \\
\text { actitudes estimulan mi aprendizaje. }\end{array}$ & 3,77 & 1,21 & \\
\cline { 2 - 5 } & $\begin{array}{l}\text { 9. Los profesores del área de ingeniería de software } \\
\text { supervisan adecuadamente mi actividad académica. }\end{array}$ & 3,80 & 1,17 & \\
\hline & Subtotal & 3,82 & 1,20 & \\
\hline
\end{tabular}

Fuente: elaboración propia 
Finalmente, el factor 4, referido a recursos físicos y virtuales, presenta el nivel más bajo de motivación (promedio 3,64), lo cual evidencia la necesidad sentida de las instituciones participantes de mejorar de forma permanente los recursos tecnológicos al servicio de los estudiantes que apoyan el desarrollo de sus competencias prácticas en el área de ingeniería de software. Ver tabla 6.

Tabla 6. Resultados del factor recursos físicos y virtuales

\begin{tabular}{|c|c|c|c|c|}
\hline \multicolumn{5}{|c|}{ Factor 4: Recursos físicos y virtuales } \\
\hline Subfactor & Ítems & Media & $\begin{array}{c}\text { Desviación } \\
\text { estándar }\end{array}$ & $\begin{array}{c}\text { Alfa de } \\
\text { Cronbach }\end{array}$ \\
\hline \multirow{5}{*}{$\begin{array}{l}\text { 4.1 Recursos físicos } \\
\text { y virtuales (RFV) }\end{array}$} & $\begin{array}{l}\text { 1. Los recursos físicos disponibles en la } \\
\text { universidad son adecuados para los aprendizajes } \\
\text { de la ingeniería del software: instalaciones, aulas, } \\
\text { laboratorios, redes, biblioteca. }\end{array}$ & 3,48 & 1,28 & \multirow{5}{*}{0,821} \\
\hline & $\begin{array}{l}\text { 2. El equipamiento disponible en los laboratorios } \\
\text { es adecuado para los aprendizajes de la ingeniería } \\
\text { del software: sistema operativo, motores de base } \\
\text { de datos, entornos de desarrollo, lenguajes de } \\
\text { programación, herramientas CASE. }\end{array}$ & 3,58 & 1,26 & \\
\hline & $\begin{array}{l}\text { 3. Los recursos virtuales, tales como campus } \\
\text { virtual, correo electrónico, biblioteca virtual } \\
\text { o servicios del portal del alumno, que me } \\
\text { proporciona la universidad, son apropiados para } \\
\text { el desarrollo de las asignaturas de la ingeniería } \\
\text { del software. }\end{array}$ & 3,82 & 1,19 & \\
\hline & $\begin{array}{l}\text { 4. Utilizo los recursos virtuales para man- } \\
\text { tener el intercambio de ideas con compa- } \\
\text { ñeros o profesores. }\end{array}$ & 3,69 & 1,29 & \\
\hline & Subtotal & 3,64 & 1,25 & \\
\hline
\end{tabular}

Fuente: elaboración propia.

En este subfactor se encontró que hay un menor nivel de motivación relacionado con los recursos físicos y virtuales en los estudiantes de niveles más avanzados del programa. Esta situación podría explicarse teniendo en cuenta que en los semestres avanzados los estudiantes ya empiezan a tener contacto con la industria de software (pasantías, proyectos integradores, trabajo parcial, docentes de la industria) y, por lo tanto, los estudiantes esperan que los entornos y herramientas que forman parte de las buenas prácticas de las empresas también puedan estar disponibles en los espacios académicos. 


\subsection{Relación entre las estrategias pedagógicas sobre la motivación en el aprendizaje de la ingeniería de software}

Las estrategias pedagógicas (subfactor 3.1) muestran poder predictivo sobre la motivación del estudiante hacia el aprendizaje de la ingeniería de software (subfactor 1.2). El análisis de regresión lineal mostró la existencia de una estrecha relación entre las variables (ecuación 2).

Ecuación 2. Relación entre motivación del estudiante y estrategias pedagógicas

$$
Y=1,31+0,68 X
$$

Donde $Y$ es el subfactor de motivación del estudiante hacia el objeto de conocimiento y $X$ representan las estrategias pedagógicas empleadas. El coeficiente de determinación R2 fue de 0,5, lo que significa que el modelo explica el $50 \%$ de la variación de la variable dependiente. El error cuadrático medio fue de 0,53.

\section{DISCUSIÓN}

\subsection{Sustentación de los ítems identificados en el trabajo}

Los ítems identificados para este trabajo están sustentados en el perfil esperado de los estudiantes de las universidades participantes que parten de una cosmovisión común [18], en el estudio de motivación de estudiantes de ingeniería [6] y en estudios que caracterizan a los ingenieros de software $[19,20]$. Desde el perfil deseado presentado por Knight [18], se consideraron los siguientes aspectos: el respeto por la individualidad, la formación integral, la importancia del trabajo en equipo (que justifica los aspectos intrínsecos del aprendiz, el trabajo en equipo y las relaciones enriquecedoras con los compañeros de clase), el papel del maestro como referente activo en la formación integral del aprendiz, la búsqueda de significado de toda experiencia y su preparación para el mundo laboral (que justifica identificar la relación con los docentes de manera separada a la calidad de la docencia y procura que las estrategias pedagógicas sean pertinentes y significativas para lograr una efectiva inserción en el mundo laboral).

Con respecto a los referentes estudiados, se hizo una cuidadosa selección de los ítems del instrumento de Fernández et al. [6], que se incluirían de manera textual (TX). Otras preguntas fueron adaptadas buscando que fueran más directas (AD) o explícitas hacia el área de ingeniería de software (AS). Otros ítems fueron redactados a partir de las características del ingeniero de software (IS) identificadas en los trabajos de Beecham et al. [19] y Hall et al. [20]. Por último, algunas preguntas fueron redactadas nuevas de acuerdo con las necesidades detectadas (NW). 
Las adaptaciones que se hicieron a las preguntas de Fernández et al. [6] en los ítems $11,12,13,15,16,28$, presentan un patrón común y estuvieron orientadas a indagar de una manera más precisa en el estudiante un aspecto particular. Por ejemplo, mientras que la pregunta 13 en el referente de partida decía "Valoro la oportunidad de influir positivamente en mis compañeros" [6] y, según el autor, se correspondía con la necesidad de poder de la teoría de McClelland, la redacción final de la pregunta en este trabajo fue: "Influyo positivamente en el aprendizaje de mis compañeros". Así redactada, la pregunta corresponde de manera más precisa con el aspecto de relación con compañeros de la teoría del factor dual de Herzberg. Otro criterio de adaptación común fue el que se aplicó en las preguntas relacionadas con los recursos físicos y virtuales $(1,2,3$ y 4$)$ y las estrategias pedagógicas $(7,8,9)$ en los cuales se adaptaron las preguntas para focalizarlas de manera específica al área de ingeniería de software. Por ejemplo, la pregunta 1 en el referente de partida decía "Los recursos físicos disponibles en la universidad son adecuados: instalaciones, aulas, laboratorios, biblioteca, transporte, etc", la cual fue adaptada de la siguiente manera: "Los recursos físicos disponibles en la universidad son adecuados para los aprendizajes de la ingeniería del software...".

Este trabajo destaca la importancia de considerar como elementos de motivación de los estudiantes de ingeniería informática los aspectos desde la perspectiva del trabajo práctico. Se comparte plenamente la premisa dada por Fernández et al. [6], en el sentido de que es importante tener en cuenta la cercanía del perfil que un estudiante de ingeniería debería tener con el perfil de un trabajador. Los aspectos de competencias técnicas $(19,17)$ se redactaron atendiendo a la segunda característica que mencionan Beecham et al. [19]. La identificación con la tarea (21, 23, 24, 25, 32, 39 y 40) se posiciona como el primer motivador general del ingeniero de software según Hall et al. [20]. La creatividad $(26,27)$ en la característica 15 se adapta de Beecham et al. [19], entre otros.

\subsection{Confiabilidad y validez del instrumento}

La idoneidad del instrumento para realizar un análisis factorial resultó excelente $(\mathrm{KMO}=0,978)$. Asimismo, la fiabilidad del instrumento, obtenido a través del Alfa de Cronbach, demuestra una alta confiabilidad de la escala de motivación tanto a nivel de factor como de subfactor. Según Mallery et al. [21], la escala es excelente $(>0,9)$ para los factores de atributos de desempeño (F1: 0,981$)$ y relación de estudiantes y docentes (F2: 0,928); y buena (entre 0,9 y 0,8$)$ para los factores de estrategias pedagógicas (F3: $0,869)$ y recursos físicos y virtuales (F4: 0,821$)$.

Vale la pena contrastar el modelo de factores y subfactores obtenido en este trabajo, con respecto al modelo de factores obtenido en el referente principal. El análisis factorial del trabajo de Fernández et al. [6] obtuvo como resultado cinco factores: 
deseo intrínseco por el desarrollo intelectual y la superación académica; elementos extrínsecos; responsabilidad y predisposición hacia el proceso de aprendizaje; definición clara de tareas y optimización del esfuerzo; participación en el proceso de selección de actividades. Estos factores explican el 45,945 \% de la varianza. En contraste, los resultados obtenidos en este trabajo a través de la matriz de factores rotados arrojaron una estructura de cuatro factores que explican el $71,5 \%$ de la varianza, sin que haya sido necesario eliminar variables para lograr la saturación, lo cual indica que se trata de un modelo con un nivel de ajuste mejor que el presentado por Fernández et al.

Desde el aspecto cualitativo, también se pueden observar algunas diferencias entre el modelo de factores obtenido por Fernández et al. [6] y el presentado en este trabajo: FF1 incluye elementos identificados en F1; FF2 agrupa elementos que tienen que ver con F2 (en lo relativo a relaciones con compañeros), F4 (en lo relativo a recursos formativos disponibles) y define algunos ítems referidos a métodos de evaluación y supervisión que no fueron incluido en este trabajo; FF3 identifica ítems que también están considerados en F1; FF4 y FF5 incluye elementos extraídos de otras teorías de motivación que no fueron considerados en este trabajo. Este análisis permite concluir que con un número menor de preguntas (cuarenta ítems) con respecto al instrumento referente (cuarenta y ocho ítems), el instrumento desarrollado en este trabajo hace un tratamiento más preciso y abarcante para estudiantes del área de informática.

La identificación de subfactores realizada de forma intencionada en la segunda fase del análisis respetó los resultados obtenidos en la primera fase. Esta identificación se realizó atendiendo principalmente al marco conceptual de enseñanza de la ingeniería de software en contexto definido en trabajos previos [16-17], en los cuales se distinguen tres dimensiones: aprendiz, cuerpo de conocimiento y contexto. Es así como el factor 1, atributos de desempeño, se refina y estructura alrededor de tres subfactores: a) motivación intrínseca del estudiante hacia el aprendizaje (MIA); b) motivación del estudiante para atender las demandas del contexto, que en este caso particular sería la industria de software (MDC); y c) su disposición para responder al objeto de conocimiento, que en este caso particular sería la ingeniería de software (MOC). El factor 2, relación con estudiantes y docentes, se descompone en subfactores que resultan obvios: a) relación con compañeros (RCO) y b) relación con docentes (RDO). Los factores 3 (estrategias pedagógicas EPE) y 4 (recursos físicos y virtuales RFV) no se descomponen por considerar que tienen el nivel de desglose adecuado para los propósitos del estudio.

El alto poder predictivo de las prácticas pedagógicas sobre la motivación del estudiante hacia el aprendizaje respalda ampliamente conclusiones obtenidas en otros estudios [22-24]. El estudio que se realizó en Colombia concluyó que la motivación 
aumenta el proceso cognitivo de forma exponencial [22]. El estudio realizado en Estados Unidos demostró que las prácticas de aprendizaje basadas en problemas son más significativas para el aprendizaje e influyen en la motivación [23]. Otro estudio de regresión lineal realizado en Estados Unidos encontró que la motivación está directamente relacionada con la realización de actividades académicas significativas y relevantes, tales como el trabajo en el aula en ambientes colaborativos, contar con tutores por asignatura, con un buen clima dentro del aula y tener una buena interacción entre estudiantes [24].

En este trabajo la obtención de factores parte del análisis de factores rotados, lo cual contrasta con trabajos que reproducen la estructura factorial de instrumentos ya validados como la prueba Motivated Strategies for Learning Questionnaire (MSLQ) [25-27].

\subsection{Acciones de mejora}

A partir de los resultados de este estudio, se han venido implementando acciones de mejora que evidencian su pertinencia, validez y proyección en el tiempo. A continuación, se sintetizan los frentes de trabajo en los cuales se está abordando:

Estrategias para mejorar la calidad de la docencia. Actualmente las universidades autoras del artículo están trabajando para definir estrategias pedagógicas transversales que mejoren el proceso enseñanza-aprendizaje, tales como aula invertida [28, 29] y coteaching [30] en las cuales se han realizado experiencias piloto en asignaturas de programación y diseño, cuyos resultados están siendo analizados.

Otra estrategia que se está fortaleciendo es el enfoque de orientación por proyectos [31]. En la reforma reciente del proyecto educativo del programa (PEP) de una de las universidades involucradas en el estudio, se definió la figura de proyecto integrador como una estrategia transversal central en la que los docentes de diferentes asignaturas asesoran y monitorean el trabajo práctico del estudiante en escenarios muy cercanos a los que se presentan en la industria. De esta manera, se busca un aprendizaje significativo y motivado por parte de los estudiantes [32, 33], dado que dichos proyectos estarían orientados a la solución de problemas reales.

Acciones para enfrentar las limitaciones de tiempo. Las restricciones de tiempo que afectan la motivación de los estudiantes encuestados es un factor externo común en las universidades vinculadas al estudio. En general, estos estudiantes viven en residencias universitarias dentro del mismo campus y deben trabajar para sufragar parte de sus gastos. Este hecho incide en la dificultad de trabajar en equipo en espacios diferentes al aula y en las limitaciones para dedicarse a otro tipo de actividades complementarias 
que fortalezcan su formación integral. Se está trabajando en la concientización a los docentes para que dosifiquen de forma equilibrada el esfuerzo que deben invertir los estudiantes fuera del aula. También se espera que los proyectos integradores contribuyan a que los estudiantes puedan capitalizar sus esfuerzos alrededor de un proyecto común que fortalezca competencias transversales comunes y competencias disciplinares de las distintas asignaturas.

\section{CONCLUSIONES}

Los altos índices de confiabilidad de la estructura de factores y subfactores, comprobada estadísticamente y por su soporte teórico, perfilan este instrumento como candidato para ser aplicado en programas de informática con énfasis en ingeniería de software en otras instituciones.

La estructura de factores y subfactores permitió identificar los aspectos positivos, los retos u oportunidades de mejora en los que deben trabajar las universidades participantes. Las acciones de mejora que se han derivado de estos resultados evidencian la pertinencia del instrumento y su potencial evolución en el tiempo.

Este trabajo respalda de manera clara la estrecha relación que existe entre las estrategias pedagógicas y la motivación del estudiante para el aprendizaje.

\section{REFERENCIAS}

[1] J. L. Contreras Véliz, J. Alanoca Gutiérrez, J. Salem Bar-Bar, J. E. Quevedo Reyes, G. Garita, R. Sepúlveda Lima, C. M. Hinojosa Raza, H. J. Duarte Pavón, A. P. Chávez Cervantes, A. E. Estrada Quintero, D. Bernal, M. E. García, A. Pow-Sang y L. González, Educación Superior en América Latina: reflexiones y perspectivas en Informática. Bilbao: Universidad de Deusto, 2013.

[2] T. C. Lethbridge, J. Diaz-Herrera, R. J. J. LeBlanc y J. B. Thompson, "Improving software practice through education: Challenges and future trends," IEEE Xplore, pp. 12-28, 2007. doi: https://doi.org/10.1109/FOSE.2007.13

[3] S. J. Martínez Marín, S. Arango Aramburo y J. Robledo Velásqiez, "El Crecimiento de la Industria del Software en Colombia: un Análisis Sistémico," Revista EIA, n. ${ }^{\circ} 23$, pp. 95-106, 2015.

[4] J. Guimón y E. Paraskevopoulou, "Factors shaping the international knowledge connectivity of industrial clusters: a comparative study of two Latin American cases," Entrepreneurship \& Regional Development, vol. 29, n. ${ }^{\circ} 9-10$, pp. 817-846, 2017. doi: https://doi.org/10.1080/08 985626.2017.1354400

[5] M. C. Rodríguez, G. E. Dabos y A. G. Rivero, "Implementação de políticas de equilíbrio trabalho-vida em pequenas e médias empresas: um estudo de casos múltiplos na indústria 
de software," Estudios gerenciales, vol. 34, n. ${ }^{\circ}$ 147, pp. 172 - 189, 2018. doi: https://doi. org/10.18046/j.estger.2018.147.2592

[6] D. L. Fernández, P. A. Cavero, M. R. Sánchez y M. L. C. Fuente, "Motivación en estudiantes de ingeniería: Un caso de estudio con teorías e instrumentos para su medida y desarrollo," REDU: Revista de Docencia Universitaria, vol. 12, n. ${ }^{\circ}$ 4, pp. 343 - 376, 2014. doi: https://doi. org/10.4995/redu.2014.5627

[7] D. López-Fernández, P. P. Alarcón y E. Tovar, "Motivation in engineering education a framework supported by evaluation instruments and enhancement resources," IEEE Global Engineering Education Conference (Educon), pp. 421-430, 2015. doi: 10.1109/ EDUCON.2015.7096006

[8] Z. Raud, V. Vodovozov, A. Aksjonov y E. Petlenkov, "Promotion of the students' success in higher engineering education," IEEE 58th International Scientific Conference on Power and Electrical Engineering of Riga Technical University (RTUCON), pp. 1-6, 2017. doi: 10.1109/ RTUCON.2017.8124806

[9] P. K. Murphy y P. A. Alexander, "A Motivated Exploration of Motivation Terminology," Contemporary Educational Psychology, vol. 25, n. ${ }^{\circ}$ 1, pp. 3-53, 2000. doi: https://doi.org/10.1006/ceps.1999.1019

[10] D. A. Bergin, M. E. Ford y R. D. Hess, "Patterns of Motivation and Social Behavior Associated With Microcomputer Use of Young Children," Journal of Educational Psychology, vol. 85, n. ${ }^{\circ}$ 3, pp. 437-445, 1993. doi: https://doi.org/10.1037/0022-0663.85.3.437

[11] P. A. Whang y G. R. Hancock, "Motivation and Mathematics Achievement: Comparisons between Asian-American and Non-Asian Students," Contemporary Educational Psychology, vol. 19, n. ${ }^{\circ}$ 3, pp. 302-322, 1994. doi: https://doi.org/10.1006/ceps.1994.1023

[12] P. R. Pintrich, "A Conceptual Framework for Assessing Motivation and Self-Regulated Learning in College Students," Educational Psychology Review, vol. 16, n. ${ }^{\circ} 4$, pp. 385-407, 2004. doi: https://doi.org/10.1007/s10648-004-0006-x

[13] B. Álvarez Álvarez, C. González Mieres y N. García Rodríguez, "La motivación y los métodos de evaluación como variables fundamentales para estimular el aprendizaje autónomo," REDU: Revista de Docencia Universitaria, vol. 5, n. ${ }^{\text {2 } 2, ~ p p . ~ 1-13, ~ 2007 . ~ d o i: ~ h t t p s: / / d o i . o r g / 10.4995 / ~}$ redu. 2007.6275

[14] A. N. Leontyev, Problems of the development of the mind. Moscú: Progress Publishers, 1981, p. 454.

[15] Y. Engeström, "Expansive Learning at Work: Toward an Activity Theoretical Reconceptualization," Journal of Education and Work, vol. 14, n. ${ }^{\circ} 1$, pp. 133-156, 2001. doi: https://doi.org/10.1080/13639080020028747

[16] R. Anaya, "Una Visión de la Enseñanza de la Ingeniería de Software como Apoyo al Mejoramiento de las Empresas de Software," Revista Universidad Eafit, vol. 42, n. ${ }^{\circ} 141$, pp. 60-76, 2006. 
[17] R. Anaya y J. Trujillo, "Un Modelo de Enseñanza en el Contexto de la Ingeniería de Software," Memorias Del IXIII Congreso Iberoamericano de Educación Superior en Computación, Pontificia Universidad Javeriana, vol. 1, n. . 1, pp. 120-132, 2005.

[18] G. R. Knight, Educación para la Eternidad: Filosofía de la educación adventista. Ciudad de México, México: GEMA Editores, 2017, p. 156.

[19] S. Beecham, N. Baddoo, H. Robinson y H. Sharp, "Motivation in Software Engineering: A systematic literature review," Information and Software Technology, vol. 50, n. 9-10, pp. 860-878, 2008. doi: https://doi.org/10.1016/j.infsof.2007.09.004

[20] T. Hall, H. Sharp, S. Beecham, N. Baddoo y H. Robinson, "What Do We Know about Developer Motivation?” IEEE Software, vol. 25, n. ${ }^{\circ}$ 4, pp. 92-94, 2008. doi: https://doi.org/10.1109/ MS.2008.105

[21] P. Mallery y D. George, SPSS/PC: Step by step: a simple guide and reference. Belmont, CA: Wadswoth Publishing Company, 1995.

[22] F. Fong Silva, J. Chiquillo Rodelo y R. Pitre-Redondo, "Mathematical Model to Characterize the Association between Cognition and Motivation in Engineering Students," Contemporary Engineering Sciences, vol. 11, n. ${ }^{\circ} 49$, pp. 2403-2412, 2018. doi: https://doi.org/10.12988/ ces.2018.83129

[23] L. C. Benson, A. Kim y B. Morkos, "Work in progress: How differences in student motivation characterize differences between engineering disciplines," Proceedings - Frontiers in Education Conference, 2012. doi: 10.1109/FIE.2012.6462358

[24] L. J. Barker, C. McDowell y K. Kalahar, "Exploring factors that influence computer science introductory course students to persist in the major," SIGCSE'09 - Proceedings of the 40th ACM Technical Symposium on Computer Science Education, pp. 153-157, 2009. doi: https:// doi.org/10.1145/1508865.1508923

[25] M. D. C. Ramírez Donantes, J. E. Canto y Rodríguez, J. A. Bueno Álvarez y A. Echazarreta Moreno, "Validación Psicométrica del Motivated Strategies for Learning Questionnaire en Universitarios Mexicanos," Electronic Journal of Research in Educational Psychology, vol. 11, n. ${ }^{\circ}$ 1, pp. 193-214, 2013. doi: http://dx.doi.org/10.25115/ejrep.v11i29.1563

[26] H. Granados López y F. A. Gallego López, “Motivación, Aprendizaje Autorregulado y Estrategias de Aprendizaje en Estudiantes de tres Universidades de Caldas y Risaralda," Revista Latinoamericana de Estudios Educativos, [En línea], vol. 12, n. . 1, pp. 71-90, Disponible: http:// latinoamericana.ucaldas.edu.co/downloads/Latinoamericana12(1)_5.pdf 2500-5324, 2016.

[27] J. Stok y J. Harari, "Student motivations as predictors of high-level cognitions in project-based classrooms," Active Learning in Higher Education, vol. 15, n. ${ }^{\circ} 3$, pp. 231-247, 2014. doi: $10.1177 / 1469787414554873$

[28] G. S. Mason, T. R. Shuman y K. E. Cook, "Comparing the effectiveness of an inverted classroom to a traditional classroom in an upper-division engineering course," IEEE Transactions on Education, vol. 56, n. ${ }^{\circ}$ 4, pp. 430-435, 2013. doi: https://doi.org/10.1109/TE.2013.2249066 
[29] T. H. C. Chian, "Analysis of learning behavior in a flipped programing classroom adopting problem-solving strategies," Interactive Learning Environments, vol. 25, n. ${ }^{\circ} 2$, pp. 189-202, 2017. doi: https://doi.org/10.1080/10494820.2016.1276084

[30] A. J. Berre, S. Huang, H. Murad y H. Alibakhsh, "Teaching modelling for requirements engineering and model-driven software development courses," Computer Science Education, vol. 28, n. ${ }^{\circ}$ 1, pp. 42-64, 2018. doi: https://doi.org/10.1080/08993408.2018.1479090

[31] M. Nauman y M. Uzair, "SE and CS collaboration: Training students for engineering large, complex systems," In 20th Conference on Software Engineering Education \& Training (CSEET'07), pp. 167-174, 2007. doi: https://doi.org/10.1109/CSEET.2007.44

[32] C. Ghezzi y D. Mandrioli, "The challenges of software engineering education," In International Conference on Software Engineering, pp. 115-127, 2005. doi: https://doi. org/10.1145/1062455.1062578

[33] M. Guzdial, "Balancing teaching CS efficiently with motivating students," Communications of the $A C M$, vol. 60, n. $^{\circ} 6$, pp. 10-11, 2017. doi: https://doi.org/10.1145/3077227 